
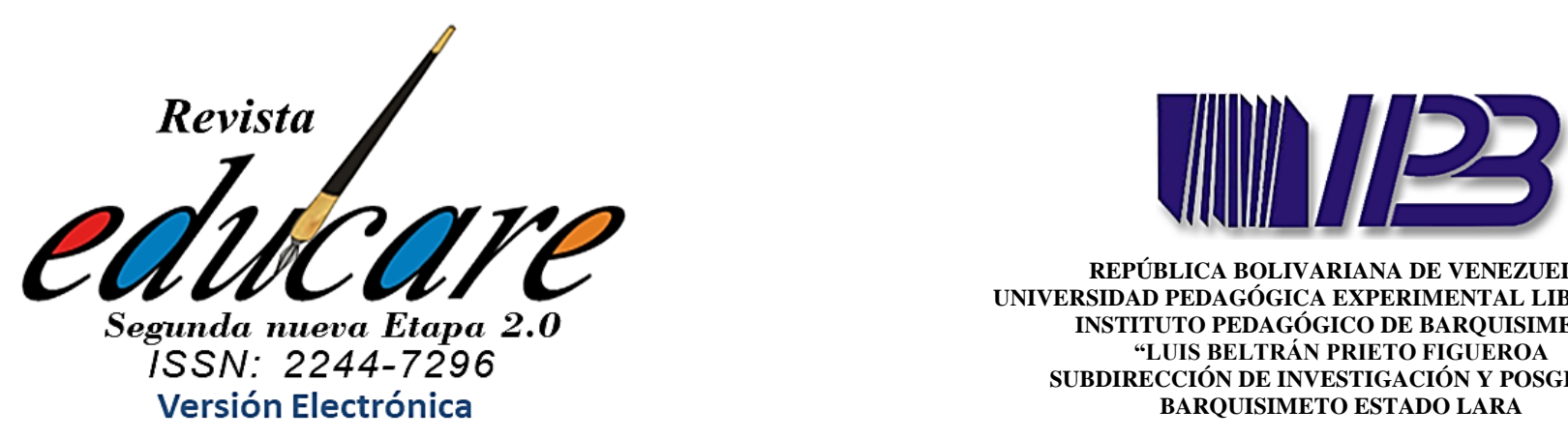

REPÚBLICA BOLIVARIANA DE VENEZUELA UNIVERSIDAD PEDAGÓGICA EXPERIMENTAL LIBERTADOR INSTITUTO PEDAGÓGICO DE BARQUISIMETO "LUIS BELTRÁN PRIETO FIGUEROA

SUBDIRECCIÓN DE INVESTIGACIÓN Y POSGRADO BARQUISIMETO ESTADO LARA

Volumen 24 № 2 Mayo-Agosto 2020

$(97-118)$

Joselyn Fuentes Aguilar *

ORCID: https://orcid.org/0000-0002-0988-0257

Francis Carolina González Pérez**

ORCID: https://orcid.org/0000-0002-2115-5576

\author{
UNIVERSIDAD PEDAGÓGICA \\ EXPERIMENTAL LIBERTADOR \\ INSTITUTO PEDAGÓGICO DE \\ BARQUISIMETO \\ VENEZUELA
}

*Profesora en la especialidad de Preescolar egresada de la Universidad Pedagógica Experimental Libertador Instituto Pedagógico de Barquisimeto (UPEL-IPB). Magister en Educación Mención Educación Inicial (UPELIPB). Docente adscrito al Centro de Educación Inicial Rosa Isabel Angulo. Correo electrónico clarielsy19@gmail.com

**Doctora en Educación (UPEL-UCLAUNEXPO). Magister en Educación Inicial (UPEL-IPB) y Profesora en Educación Preescolar (UPEL-IPB). Docente categoría Agregado en la UPEL-IPB en pregrado y posgrado. Coordinadora de Promoción y Difusión de la Investigación (UPEL-IPB). Coordinadora de la línea de Investigación: "Educación Infantil en Contextos Sociocomunitarios". Correo electrónico: prof.franciscarolinagonzalez@gmail.com -Este artículo se inserta en la línea de investigación: "Saber Pedagógico del Profesional Docente de Educación Infantil (UPEL-IPB).

\section{ACTITUD DE LAS DOCENTES HACIA LA ATENCIÓN PEDAGÓGICA DEL NIÑO EN LA ETAPA MATERNAL}

\author{
ATTITUDE OF TEACHERS TOWARDS THE \\ PEDAGOGICAL CARE OF THE CHILD IN THE \\ MATERNAL STAGE
}

Recibido:

15-12-2019

Aceptado:

30-04-2020 

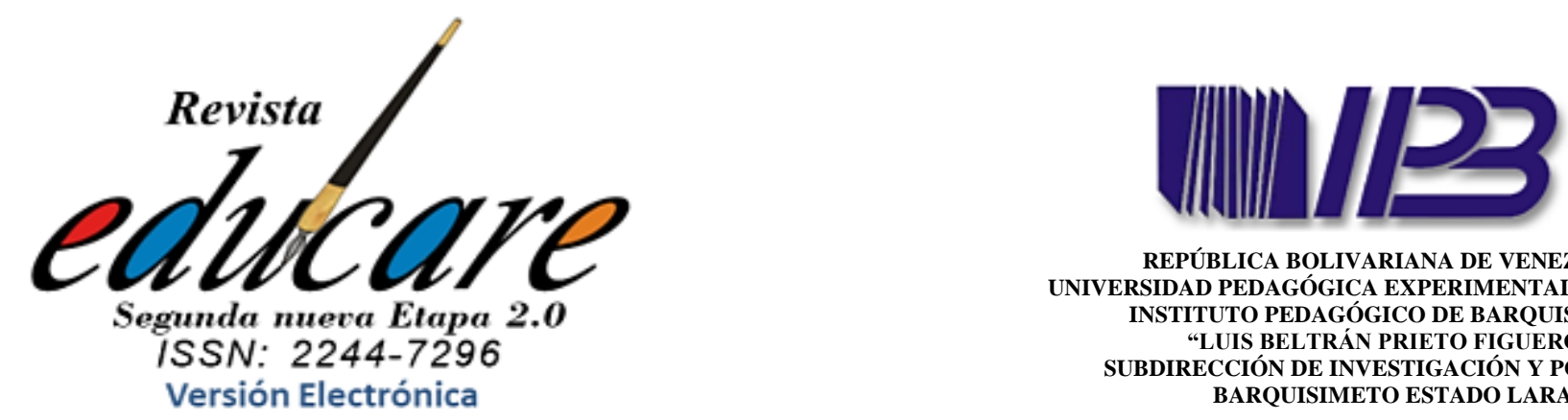

REPÚBLICA BOLIVARIANA DE VENEZUELA UNIVERSIDAD PEDAGÓGICA EXPERIMENTAL LIBERTADOR INSTITUTO PEDAGÓGICO DE BARQUISIMETO "LUIS BELTRÁN PRIETO FIGUEROA

SUBDIRECCIÓN DE INVESTIGACIÓN Y POSGRADO BARQUISIMETO ESTADO LARA

\section{ACTITUD DE LAS DOCENTES HACIA LA ATENCIÓN PEDAGÓGICA DEL NIÑO EN LA ETAPA MATERNAL}

ATTITUDE OF TEACHERS TOWARDS THE PEDAGOGICAL CARE OF THE CHILD IN THE MATERNAL STAGE

\title{
Resumen
}

La presente investigación tuvo como objetivo analizar la actitud de las docentes de Educación Inicial hacia la Atención Pedagógica en la Etapa Maternal en los C.E.I. de la Parroquia Mariano Peraza del Municipio Jiménez estado Lara. Metodológicamente se basa en un enfoque cuantitativo, enmarcada en un diseño exploratoriodescriptivo. Los sujetos de estudio fueron treinta (30) docentes a los cuales se les aplicó un cuestionario de 34 ítems con opciones de respuesta en escala tipo Likert, validado a través de un juicio de expertos y con una confiabilidad según el estadístico Alpha de Cronbach de 0,95 que representa una alta confiabilidad. Los resultados fueron procesados por medio de la estadística descriptiva y se concluyó que existen dicotomías que caracterizan las dimensiones cognitiva, afectiva y conductual de la actitud docente hacia la atención a los niños de 0 a 3 años, entre una actitud favorable y desfavorable.

Palabras clave: actitud, docentes, etapa maternal, atención pedagógica.

\begin{abstract}
The objective of this research was to analyze the attitude of Initial Education teachers towards Pedagogical Attention in the Maternal Stage in the C.E.I. from the Mariano Peraza Parish in the Jiménez Municipality, Lara state. Methodologically it is based on a quantitative approach, framed in an exploratory-descriptive design. The study subjects were thirty (30) teachers to whom a 34-item questionnaire with response options on a Likert-type scale was applied, validated through expert judgment and with a reliability according to the Cronbach Alpha statistic of 0 , 95 representing high reliability. The results were processed using descriptive statistics and it was concluded that there are dichotomies that characterize the cognitive, affective and behavioral dimensions of the teaching attitude towards the attention to children from 0 to 3 years old, between a favorable and unfavorable attitude.
\end{abstract}

Keywords: attitude, teachers, maternal stage, pedagogical attention. 


\section{INTRODUCCIÓN}

Existe un creciente interés mundial por el desarrollo y aprendizaje de la infancia, el cual se ha concretado en lo que se ha denominado Atención y Educación de la Primera Infancia (AEPI). Según la Organización de las Naciones Unidas para la Educación, la Ciencia y la Cultura (UNESCO, 2007), los primeros años de vida del niño contemplan un desarrollo cerebral excepcional que sirve de base a los aprendizajes siguientes y requieren una atención y educación específica sustentada en el apoyo cognitivo-afectivo de quienes lo atienden.

En el contexto venezolano, la atención educativa a la primera infancia corresponde al nivel de Educación Inicial definido como la atención integral a niños y niñas desde su concepción hasta cumplir los 6 años de edad. De acuerdo al Ministerio de Educación y Deportes (MED, 2005) comprende dos etapas: Maternal (0-3 años) y Preescolar (3-6 años). En el caso de Maternal, la Constitución Nacional (1999) lo designa como nivel educativo y por primera vez establece su obligatoriedad junto a los otros niveles.

La reconfiguración de la educación Inicial en el contexto venezolano, y la incorporación de la etapa maternal constituyó una verdadera transformación en el sistema educativo en cuanto a la concepción de la condición de educabilidad del niño más pequeño, pues hasta ese momento, los niños de estas edades eran atendidos por Madres Cuidadoras en Hogares de Cuidado desde la denominada Atención No Convencional (no formal) en programas dirigidos a los niños con menos recursos y excluidos del sistema educativo.

Es así como en el año 2002 se elabora la propuesta del Currículo Básico Nacional de Educación Inicial en la que se hace una primera aproximación operativa del maternal y preescolar acorde a las políticas nacionales y los compromisos internacionales adquiridos. Ya en el año 2005 se produce el documento curricular vigente denominado Currículo de Educación Inicial (Ministerio de Educación y Deportes, MED, 2005) que establece a la Educación Inicial como etapa a Maternal y Preescolar como niveles en el sistema educativo. Sin embargo, se producen una serie de tensiones en la denominación de los mismos, pues en el año 2009 en la Ley Orgánica de Educación se incorpora la Educación Inicial como nivel y Maternal y Preescolar como etapas. Según el currículo de Educación Inicial (MED, 2005), la etapa Maternal (0-3 años) enfatiza la educación integral y el cuidado amoroso que proveen tanto las familias como los docentes que atienden a los infantes, pues éstos requieren establecer vínculos 
nutritivos con las personas que le rodean y desarrollar relaciones de seguridad y confianza a través del contacto humano afectivo, dialogante y respetuoso para potenciar su desarrollo físico, emocional, cognitivo y social y así desarrollar relaciones de seguridad y confianza (New Jersey Council For Young Children, 2013). Así mismo, es importante mencionar que según Narodowski y Snaider (2017) las categorías de diferenciación de los niños de 0 a 3 años se producen según las características de su desarrollo evolutivo: los que toman leche materna o lactantes y los que se desplazan de forma autónoma o deambuladores.

La incorporación de la atención pedagógica del niño de 0 a 3 años a los currículos latinoamericanos plantea de igual manera un reto, ya que de acuerdo a Peralta (2007) se ha hecho una transición del concepto de "estimulación temprana" propio del área de la salud hacia una concepción pedagógica de la educación formal de esta franja etaria que signifique socialmente la necesidad de una "labor verdaderamente educativa en los primeros años de vida" (p. 2). En Venezuela, se incluye además en la etapa maternal los procesos formativos y acciones orientadoras a madres embarazadas relacionados a el desarrollo integral del niño, es decir, la etapa maternal se concreta en espacios convencionales o Centros de Educación Inicial (CEI) y, en espacios de atención no convencional en las comunidades atendidos por madres integrales en los Simoncitos Comunitarios (González, 2019).

Sin embargo, ¿cómo se ha llevado a cabo esta atención? ¿Ha podido la docente de educación inicial asumir este nuevo reto educativo? ¿Cuál ha sido su actitud al respecto? Es menester, ubicar el contexto del cual surgen estas interrogantes pues una de las autoras de esta investigación como docente de la Universidad Pedagógica Experimental Libertador Instituto Pedagógico de Barquisimeto (UPEL-IPB) y tutora de la práctica profesional en la especialidad de Educación Inicial mantiene una constante presencia en los centros educativos. En estos, se ha podido constatar a través de conversaciones informales cierta resistencia de las maestras a ser asignadas como docentes en el aula de maternal, por lo cual los directivos suelen mostrarse muy preocupados por las manifiestas dudas que existen entre el equipo docente ante la incorporación de aulas de maternal al centro, ya que las comunidades comúnmente solicitan más apertura de aulas debido a la cantidad de niños existentes.

Asimismo, la experiencia académica ha permitido evidenciar que la labor pedagógica en esta etapa presenta características similares a las estrategias pedagógicas que se desarrollan en el preescolar e incluso a los contenidos trabajados en forma de aprendizajes esperados con una tendencia a "preescolarizar" la etapa: reconocimiento de colores, números y formas

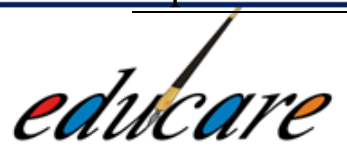


geométricas, la utilización de dibujos prediseñados y una disminución de los juegos y actividades corporales tan necesarias en estas edades ( 0 a 3 años). Como expone Pares (2012) un docente debe conocer el desarrollo infantil desde un enfoque contextual porque el aprendizaje y el desarrollo resultan de la combinación de las estructuras biológicas con la mediación sociocultural, asimismo los docentes deben comprender las necesidades e intereses de los niños y ajustar su práctica pedagógica para generar pertinencia y calidad en los aprendizajes.

Otro reto para el análisis ha surgido a partir de la revisión documental, la cual deja entrever que son escasos los trabajos empíricos acerca de la relación educativa en el maternal y del rol del docente en este ámbito, situación que pudiera crear nuevas perspectivas pedagógicas y construir y reconstruir saberes en cuanto a la educación de la infancia en edades de 0 a 3 años y que le da mayor pertinencia a este estudio. Molina, Sendra y Sierra (2016) argumentan que el docente de infantil debe aprender que está en una relación de alteridad y en consecuencia puede crear saberes a partir de las tradiciones pedagógicas e incluso de los saberes disciplinares disponibles.

Las consideraciones anteriores, pudieran ser indicativo de la necesidad de desarrollar nuevos estudios sobre el tema para que desde la formación pedagógica y la gestión educativa se puedan aplicar correctivos a dicha situación, pues surgen diferentes interrogantes ¿Existe una adecuada cualificación del docente venezolano para trabajar con las edades de 0 a 3 años? ¿Cuál es la actitud del docente ante este nuevo reto? La actitud se asume en este estudio como la disposición, aceptación o rechazo hacia la atención pedagógica en la etapa maternal, considerando los tres componentes: cognitivo, afectivo y conductual, pues como plantea Zubieta (2005) la actitud expresa los valores de la persona y le permite manifestar lo que conoce y siente respecto a algún aspecto de interés. En consecuencia, el objetivo planteado en este estudio es: Analizar la actitud de las docentes de Educación Inicial hacia la Atención Pedagógica en la Etapa Maternal. 


\section{Fundamentos para la atención pedagógica de 0 a 3 años}

La inserción formal de la etapa maternal en el sistema educativo venezolano en el año 2005, constituye una política trascendente desde dos ámbitos comprensivos: 1) en cuanto a la concepción del niño y su educación, puesto que se fundamenta en el potencial de aprendizaje infantil y su condición de sujeto social poseedor de características particulares que requieren una educación particular; 2) en cuanto al reconocimiento pedagógico de la atención a las edades de 0 a 3 años en la sociedad, al brindar espacios de formación, juego e interrelaciones vitales para el desarrollo infantil.

$\mathrm{Al}$ respecto, diferentes autores han establecido la importancia de educar a los niños en los tres primeros años de vida y de proveer ambientes adecuados para generar un impacto positivo y potenciador en el desarrollo y aprendizaje (Castro y Morales, 2015; Gutiérrez y Ruiz, 2018). Este nuevo posicionamiento ontoepistémico con respecto a la educación de los más pequeños busca además romper con la representación social subyacente acerca de la poca capacidad de los niños en estas edades para permanecer en una jornada escolar y poder desenvolverse con cierta autonomía junto a los otros. Sobre esto, Pitluk (2014) menciona: "es común escuchar que "los/as niños/as en estas edades no pueden intercambiar ni realizar interacciones", sin embargo los podemos observar comunicándose corporalmente, atentos unos a los otros, mirándose, tocándose, quitándose o prestándose los objetos” (p. 18).

Fundamentar la atención y educación del niño de 0 a 3 años puede hacerse en tres perspectivas de análisis: los aportes de las neurociencias, los derechos humanos y la condición de educabilidad del niño (lo pedagógico). Primeramente, es importante mencionar que durante los tres primeros años de vida se fundan las funciones cerebrales, en consecuencia los cambios cerebrales que allí ocurren modelan y regulan las habilidades, destrezas y aptitudes que continuarán en desarrollo a lo largo del ciclo vital (Amar et al., 2016).

Desde la perspectiva de las neurociencias, la plasticidad cerebral o capacidad del cerebro para modificarse está acompañada por un período sensible y por ende en estas edades se necesitan experiencias claves que activen las conexiones sinápticas requeridas para "desarrollar verdaderos bosques neuronales, que serán la base de patrones y esquemas mentales necesarios para el aprendizaje escolar de la educación primaria" (Paniagua, 2016, p. 85). En este sentido, se puede afirmar que el individuo no está signado por un determinismo biológico 
cerebral sino que a partir de la relación con el medio se puede potenciar su aprendizaje y desarrollo, sobre todo en la primera infancia.

Con respecto a la perspectiva de derechos, en la actualidad se considera al niño como un sujeto pleno de derechos y en Venezuela los artículos 102 y 103 de la Constitución Nacional asumen la educación como derecho humano y deber social fundamental, además de su obligatoriedad en igualdad de oportunidades desde el maternal. Esta postura es cónsona con lo declarado en la Convención de los Derechos del Niño en la Asamblea General de las Naciones Unidas (ONU, 1989), la cual explicita su derecho a la educación y el interés superior del niño como doctrina rectora en los países que lo suscribieron, y así pasar de una representación de un niño necesitado y carente de potencialidades a una infancia de derechos y capacidades.

Asimismo, desde la perspectiva pedagógica se reconoce la condición de educabilidad de los niños, mismos que muestran un gran interés por explorar, moverse y conocer el mundo que los rodea. Obviamente, esta potencialidad natural en los niños requiere también una mirada amplia y constructivista del aprendizaje infantil de parte del docente y los adultos significativos que le atienden, por ende, en la etapa maternal el juego, el movimiento y la afectividad son ejes pedagógicos claves al proporcionar experiencias pedagógicas significativas a los niños. Bredekamp y Coople (2006, citado por Tovar, Pastor y Lemus, 2011) identifican algunos principios de desarrollo y aprendizaje infantil que pueden orientar las prácticas educativas en estas edades, entre ellos:

- El carácter interrelacional e integrador de las áreas de desarrollo, que se dividen de manera arbitraria para la comprensión del individuo pero que en la realidad se manifiestan como un todo indivisible: las áreas de lenguaje, cognitivo, emocional y corporal están ineludiblemente relacionadas en el ser humano.

- Existe una diferenciación individual en el ritmo de aprender y desarrollarse propio de cada niño. Por ejemplo: un niño de 2 años puede mostrar una pronunciación de las palabras más clara que otro niño de la misma edad.

- Hay un efecto acumulativo y a largo plazo en las experiencias educativas que se le brindan a los niños, lo que requiere el conocimiento del docente y adultos significativos acerca del desarrollo infantil para promoverlo adecuadamente.

- El contexto sociocultural es vital para el desarrollo y aprendizaje, no solo porque amplía su visión progresiva del mundo sino porque aprende a establecer relaciones 
con los otros y a conocer los patrones socioculturales que configuran continuamente su identidad.

- El desarrollo y aprendizaje del niño se produce a partir de los elementos biológicos y ambientales.

- El juego es el mejor método de enseñanza en esta etapa.

- Es importante ofrecer seguridad física y psicológica al niño, pues esto garantiza la satisfacción de sus necesidades básicas.

Así, la educación del niño maternal crea un sinfín de oportunidades en su vida, aprovechando la sensibilidad de su desarrollo y las características de flexibilidad y apertura hacia el mundo que le rodea, pues el niño demanda que se le trate, cuide y eduque como un auténtico ser humano, con respeto y amor.

\section{Perfil y actitud del docente}

En atención a lo señalado, es de especial interés el perfil y la actitud del docente de educación inicial hacia la atención de los niños de maternal pues sus características particulares y necesidades vitales requieren docentes con las competencias necesarias para brindar atención integral, oportuna y de calidad desde el mismo momento de la gestación. Por ello, el docente a cargo de los de los niños (as) de la Etapa Maternal debe conocer y valorar su desarrollo evolutivo, que le permita comprender de sus necesidades, intereses y de los requerimientos propios de éstos para lograr un sano desarrollo de la personalidad, que pueda relacionarse proactivamente con las familias y orientar el proceso educativo, ya que el docente posee lo que llama Fujimoto (2010) un "protagonismo social, de liderazgo de los programas, de respeto a los valores culturales de las comunidades (...) Su trabajo pedagógico es con adultos, con niños, con otros agentes y con la comunidad" (p. 114).

El docente de maternal debe atender la especificidad propia de las edades de los niños (as) de 0 a 3 años y mantener una vinculación con su entorno familiar a fin de consensuar aspectos fundamentales de la enseñanza y procurar la continuidad de los aprendizajes en el hogar y en la escuela. Al respecto, Picco y Soto (2013) señalan que la educación de infantes entre 0 y 3 años requiere la vinculación de la familia y la escuela de la formación relacionada a las pautas de crianza (hábitos, pautas sociales), los aspectos de desarrollo infantil que pueden ser mediados a través de la participación guiada en los espacios en los cuales se desarrollan las 
interacciones y la consideración de los aspectos culturales para la implementación de las actividades pedagógicas en el aula (pintar, cantar, bailar, otras).

Para ello, el docente tendría que poseer una actitud receptiva no solo a la acción pedagógica y al conocimiento requerido para atender las especificidades propias de las edades tempranas, sino a la incorporación directa de la familia en el proceso educativo. Al respecto, la adaptación escolar, las actividades didácticas pertinentes, los rasgos emocionales del docente, entre otras, son saberes y haceres necesarios para que éste pueda atender a los niños de maternal, prácticas que pueden generar ciertas que tensiones en los docentes por las distintas formas de ser de los infantes (Ospina, 2015).

Sobre este aspecto, se encontró en la indagatoria documental un estudio de Saldivia (2014) acerca de la actitud del docente ante la aplicación de estrategias de integración para el proceso de adaptación en el niño del nivel maternal el cual pudo determinar a través de una muestra intencionada conformada por veinte (20) docentes que éstos tienen una actitud desfavorable en cuanto al conocimiento, creencias, sentimientos y comportamiento ante la aplicación de estrategias de integración para el proceso de adaptación en el niño de la etapa maternal, incluso detectó algunas carencias en la praxis de las docentes que laboran en el maternal pues el 50\% de los docentes desconocen las concepciones y aplicabilidad de las estrategias de integración para el grupo de 0 a 3 años.

A los efectos de estos planteamientos, el MED (2005) ha establecido tres dimensiones del perfil que debe poseer un docente de educación inicial: a) Dimensión personal, vinculada con el ser del docente y que da cuenta de sus características y rasgos personales para poder atender a los niños (salud física, cuidado personal, estabilidad emocional, afectividad); b) Dimensión pedagógica - profesional, relacionada al conocimiento de la cultura general y a los saberes específicos acerca de la educación a niños en la primera infancia; c) Dimensión socialcultural relacionada con el aprender a convivir, que responde a la participación y cooperación con los demás en todas las actividades de la vida humana.

De allí, que una actitud positiva hacia la formación de los niños es fundamental para una labor pedagógica significativa y de impacto que ayude a sentar las bases de su aprendizaje. Rodríguez (2006) define la actitud como "una organización duradera de creencias y cogniciones en general, dotada de una carga afectiva a favor o en contra de un objeto definido, que predispone a una acción coherente con las cogniciones y afectos relativos a dicho objeto" (p.8). 
Afirma el autor que los componentes cognoscitivos, afectivos y los relacionados a la conducta que incluyen las actitudes sociales, pueden modificarse a partir de cambios en uno de ellos. El componente cognoscitivo está compuesto por los conocimientos y elementos de índole cognitivo y se relacionan con la representación mental de la situación u objeto que tenga el sujeto. En el caso de la educación a niños de 0 a 3 años, el docente requiere el manejo teorético acerca del desarrollo y aprendizaje infantil, de las características del niño y de algunos postulados psicológicos y pedagógicos para potenciar el proceso de aprendizaje.

El componente afectivo de la actitud, está relacionado con los sentimientos, expresiones y emociones de la persona, la cual realiza una valoración emocional positiva o negativa que le producen un efecto de agrado o desagrado ante algo. Se considera que todo el personal docente de Educación Inicial que trabaja con la atención hacia niños de 0 a 3 años, además de su formación académica debe atender al niño con respeto y una carga afectiva importante que le demuestre a éste comprensión, la ternura y cariño. En relación al componente conductual, se refiere a cómo la persona se comporta situaciones específicas ante el objeto actitudinal en este caso la actitud hacia la atención pedagógica maternal.

En este propósito, un docente de maternal debe poseer una formación que le permita fortalecer el desarrollo de las potencialidades del niño y la niña, lo que se logra a través de una adecuada mediación de los aprendizajes; es necesario que conozca, el desarrollo evolutivo del niño y la niña, debe estar preparado para desarrollar su labor en diferentes espacios tanto convencionales como no convencionales en cualquier comunidad, siempre pensando en el beneficio de ellos/as.

\section{METODOLOGÍA}

La investigación presentada se inserta en una metodología cuantitativa, definida como "La investigación que predominantemente tiende a usar instrumentos de medición y comparación que proporcionan datos cuyo estudio requiere el uso de modelos matemáticos y de la estadística" (Hurtado y Toro, 2007, p. 41).

En cuanto al tipo de investigación, se trata de un estudio de campo de carácter exploratorio-descriptivo. Según Sabino (2007) es de campo porque los datos son recogidos directamente de la realidad estudiada, en este caso, en los Centros de Educación Inicial de una parroquia del Estado Lara, Venezuela. A su vez, constituye un estudio exploratorio por la ausencia de información con respecto a la actitud del docente en la etapa maternal y los escasos 
estudios relacionados a la atención pedagógica de 0 a 3 años. Es un estudio descriptivo porque persigue la medición o recolección de datos (Hernández, Fernández y Baptista, 2003) acerca de la variable actitud especificando sus propiedades o componentes: cognitivo, afectivo y conductual. Dicha variable fue operacionalizada de la siguiente manera:

\section{Tabla 1}

Operacionalización de la variable

\begin{tabular}{cccc}
\hline Variable & Dimensiones & Indicadores & Ítems \\
\hline & Cognitiva & Atención pedagógica & $1,2,3,4,5,6,7,8$ \\
& & Ambiente de aprendizaje & $9,10,11,12,13,14,15$, \\
Actitud de las & & & $16,17,18,19,20,21$ \\
$\begin{array}{c}\text { Docentes de } \\
\text { Educación Inicial } \\
\text { hacia la atención } \\
\text { pedagógica del } \\
\text { niño 0 a 3 años }\end{array}$ & Afectiva & Agrado & $22,23,24,25$ \\
\cline { 2 - 4 } & Conductual & Demostración de afecto & $26,27,28$ \\
& & Disposición & $29,30,31$ \\
& & Atención & $32,33,34$ \\
\hline
\end{tabular}

Nota: Elaboración propia

Población y muestra: La muestra seleccionada para este estudio es de treinta (30) docentes, que representan la totalidad de la población, es decir, todos los docentes de la etapa maternal de los Centros de Educación Inicial de la Parroquia Mariano Peraza del Municipio Jiménez, situada en una zona agrícola del Estado Lara.

Recolección de datos: Se utilizó la técnica de la encuesta y como instrumento el cuestionario. El mismo es contentivo de 34 ítems redactados en forma de afirmaciones para calificar la actitud del docente, variable que se está midiendo (Hernández, Fernández y Baptista, 2006). Cada ítems consta de 5 alternativas de respuesta con escala tipo Likert, específico para medir la actitud: Totalmente de Acuerdo (TDA), De Acuerdo (DA), Indeciso (I), En Desacuerdo (EDA), Totalmente en Desacuerdo (TED). Cabe mencionar, que los ítems corresponden a los requerimientos establecidos por el Ministerio de Educación y Deportes (2005) en cuanto a la práctica pedagógica en la etapa maternal.

Validez y Confiabilidad: En el caso particular de esta investigación, la validez del instrumento se determinó recurriendo al juicio de tres (3) expertos en el área de especialidad 
que además son investigadores universitarios acreditados. Los criterios de validación fueron: claridad, congruencia y pertinencia con los objetivos de investigación, y se solicitó sugerencias a cada ítems que permitiera “dejar, modificar o eliminar" el ítems evaluado. Por otra parte, para llevar a efecto el cálculo de la confiabilidad del instrumento a utilizar en la investigación se aplicó una prueba piloto a diez (10) docentes que no formaron parte de la muestra de estudio, pero presentan las mismas características, lo cual permitió mejorar la redacción de los ítems. Se determinó mediante la aplicación de la fórmula Alfa de Cronbach un coeficiente de $\alpha=0,95$ que representa una alta confiabilidad del instrumento diseñado.

Análisis de datos: Se empleó la estadística descriptiva, a través de la determinación de las frecuencias relativas y porcentajes de las respuestas de los docentes. Para ello, se diseñó una matriz de doble entrada en Excel donde se asentaron las respuestas suministradas en cada ítem. Posteriormente, se presentaron en gráficos con sus respectivos análisis. Luego, los datos se confrontaron con algunos aportes teóricos que se consideraron pertinentes de acuerdo a los resultados obtenidos.

\section{RESULTADOS}

En este apartado, se muestran los resultados de la aplicación del cuestionario a las docentes en ejercicio acerca de la atención pedagógica a los niños de 0 a 3 años, organizada en 3 dimensiones: cognitiva, afectiva y conductual:

\section{Dimensión cognitiva}

Los resultados revelan resultados parecidos en cuanto a la actitud desfavorable de las docentes hacia el conocimiento, identificación y reconocimiento de aspectos relacionados al desarrollo y aprendizaje infantil en edades de 0 a 3 años. Los valores porcentuales más altos en la escala de actitud corresponden a un $73 \%$ totalmente en desacuerdo en el reconocimiento de características de los niños de maternal más pequeños (de 0 a 1 año) e igualmente un $73 \%$ totalmente en desacuerdo con manejar los fundamentos pedagógicos y psicológicos para la atención pedagógica en esta etapa. Por otra parte, la actitud hacia el conocimiento de las características, necesidades e intereses de los niños de 0 a 3 años, también es desfavorable: un $60 \%$ se encuentra totalmente en desacuerdo con esta afirmación y un $23 \%$ indeciso en su respuesta (Tabla 2). 


\section{Tabla 2}

Respuestas de los docentes en cuanto a la Dimensión cognitiva, Indicador Atención pedagógica.

\section{ÍTEM}

D

ED

1 Pienso que el desarrollo y ejecución de estrategias en la etapa maternal son de formación, orientación y modelaje.

2 Conozco las características del desarrollo infantil de 0 a 3 años, $\quad \begin{array}{llllllllllll}2 & 2 & 7 & 1 & 3 & 7 & 23 & 2 & 7 & 18 & 60\end{array}$ considerando los intereses y necesidades básicas potencialidades del niño.

3 Identifico aspectos importantes del desarrollo cerebral de un niño de
5 0 a 3 años para llevar a cabo su práctica pedagógica ajustada a nuevas tendencias educativas.

4 Conozco el desarrollo del apego infantil, para su manejo apropiado. $\quad \begin{array}{lllllllllll}6 & 20 & 5 & 17 & 3 & 10 & 4 & 13 & 12 & 40\end{array}$

5 Reconozco las características del desarrollo evolutivo relacionadas $\quad \begin{array}{lllllllllll}2 & 7 & 2 & 7 & 0 & 0 & 4 & 13 & 22 & 73\end{array}$ con la alimentación del niño entre 0 y 1 años

6 Identifico las características del desarrollo evolutivo relacionadas $\quad \begin{array}{lllllllllll}1 & 3 & 6 & 20 & 2 & 7 & 2 & 7 & 19 & 63\end{array}$ con la alimentación del niño entre 1 y 3 años

7 Manejo las orientaciones generales que todo adulto significativo $\quad \begin{array}{llllllllll}0 & 0 & 0 & 4 & 13 & 4 & 13 & 3 & 11 & 19\end{array}$ debe tomar en cuenta con relación a la alimentación del niño maternal.

8 Manejo los aportes teóricos de Erickson, Piaget y Bowlby como fundamentos psicológicos y pedagógicos de la etapa maternal.

Nota: Elaboración propia.

Acerca de los resultados relacionados al indicador ambiente de aprendizaje (tabla 3) se observa que las respuestas se ubican mayormente hacia la respuesta totalmente en desacuerdo hacia las afirmaciones presentadas. Un $73 \%$ de las docentes responde estar totalmente en desacuerdo con conocer las características del ambiente físico del aula de maternal. Solo un 3\% asegura estar de acuerdo en reconocer cuáles materiales didácticos son requeridos de 0 a 1 año y un $5 \%$ en los de 1 a 2 años y 2 y 3 años.

Por otra parte, haciendo una sumatoria de las respuestas entre indeciso, en desacuerdo y totalmente en desacuerdo ante la organización de un aula de maternal la puntuación es notable, un 67\% de las docentes (ítem 15), lo que alude a una actitud que tiende a ser desfavorable hacia la misma. Otro aspecto relevante es la organización temporal de la jornada diaria de clases en maternal pues solo un 17\% se encuentra totalmente de acuerdo de saber hacerlo y otro $17 \%$ de acuerdo; siendo la jornada un elemento clave para el trabajo con niños pequeños ya que por su carácter predictivo para el niño le da sensación de seguridad y estabilidad en el ambiente escolar (MED, 2005). 
Tabla 3

Respuestas de los docentes en cuanto a la Dimensión Cognitiva. Indicador: Ambiente de Aprendizaje.

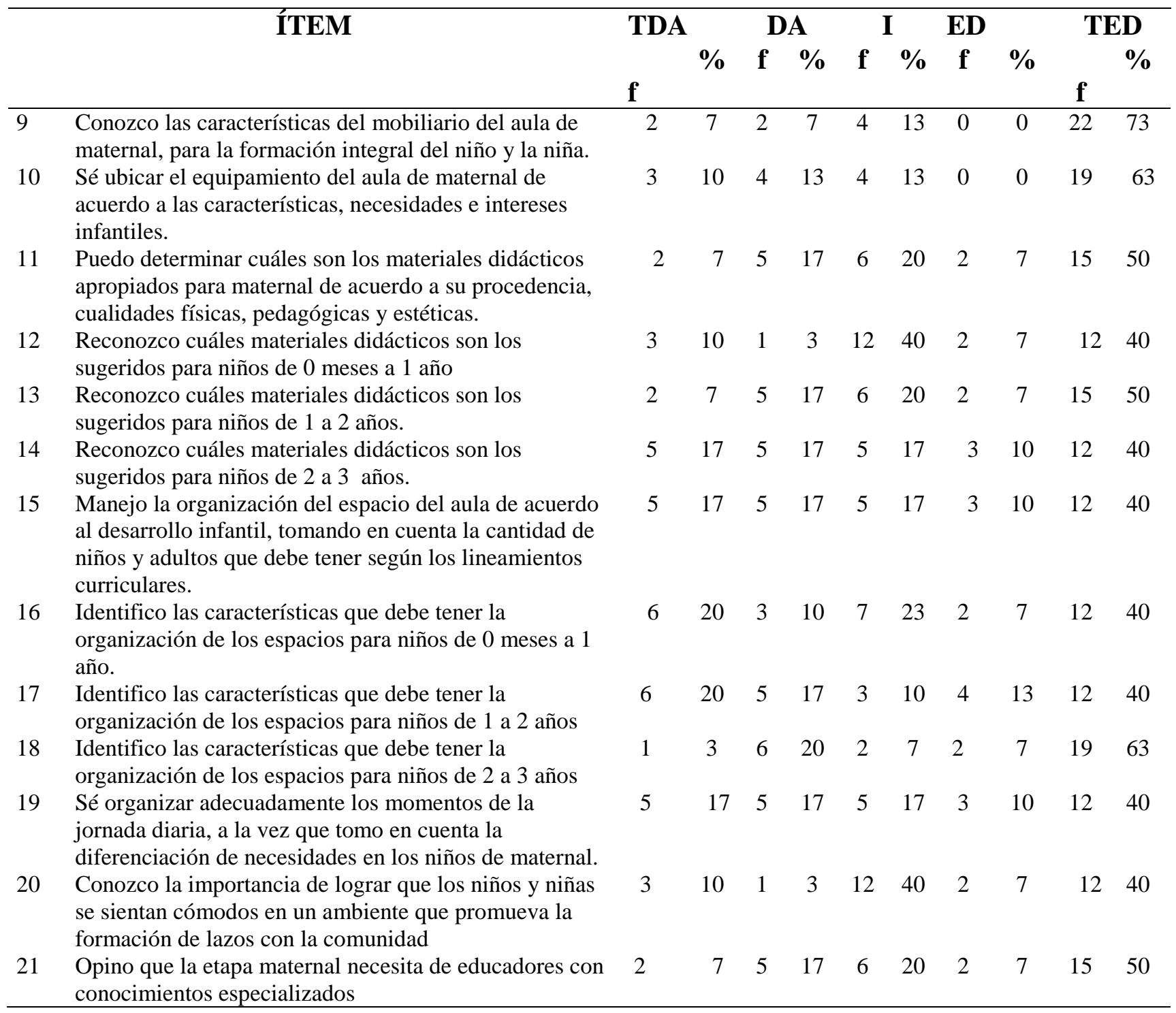

Nota: Elaboración propia.

\section{Dimensión Afectiva}

En cuanto a las respuestas relacionadas a la dimensión afectiva de la actitud de las docentes (tabla 4) aunque el porcentaje de agrado hacia el trabajo con niños de maternal es bajo si se observan los ítem 22 y 23, parece tener más aceptación el trabajo con grupo de niños de 1 a 2 años que con los bebés ( 0 a 1 año): $10 \%$ totalmente de acuerdo con la primera afirmación y 
7\% de acuerdo con la segunda. Por otra parte, se muestra que el $30 \%$ está totalmente de acuerdo en que le desagrada hacer aseo personal a los niños del aula (ítem 24) y un 30\% rechaza atender al niño en sus necesidades fisiológicas básicas pues no corresponde a su rol de educadora, junto a un $20 \%$ que se encuentra también de acuerdo con esta afirmación. Esta aseveración es sustentada por Echenique y Fermín (2011) quienes aseguran que los docentes venezolanos "no poseen orientaciones pedagógicas claras que les permitan ofrecer una educación oportuna y pertinente desde una visión integral, es decir, para ellos la atención a las necesidades vitales y las acciones pedagógicas se encuentran separadas" (p. 153).

\section{Tabla 4}

Respuestas de las docentes en cuanto a la Dimensión Afectiva, Indicador Agrado.

\begin{tabular}{|c|c|c|c|c|c|c|c|c|c|c|c|}
\hline \multirow{2}{*}{\multicolumn{2}{|c|}{ ÍTEM }} & \multicolumn{2}{|c|}{ TDA } & \multicolumn{2}{|c|}{ DA } & \multicolumn{2}{|r|}{ I } & \multicolumn{2}{|c|}{ ED } & \multicolumn{2}{|c|}{ TED } \\
\hline & & $\mathbf{f}$ & $\%$ & $\mathbf{f}$ & $\%$ & f & $\%$ & f & $\%$ & f & $\%$ \\
\hline 22 & $\begin{array}{l}\text { Me agrada trabajar con niños de edades entre } 0 \text { y } 1 \text { año, porque me permite } \\
\text { atender sus aprendizajes, necesidades, intereses y sus logros. }\end{array}$ & 2 & 7 & 5 & 17 & 6 & 20 & 2 & 7 & 15 & 50 \\
\hline 23 & $\begin{array}{l}\text { Disfruto el trabajo pedagógico con niños entre } 1 \text { y } 2 \text { años, porque me } \\
\text { permite proporcionarle experiencias significativas para alcanzar el } \\
\text { desarrollo de sus potencialidades. }\end{array}$ & 3 & 10 & 0 & 0 & 9 & 30 & 3 & 10 & 15 & 50 \\
\hline 24 & $\begin{array}{l}\text { Me desagrada realizar aseo personal a los niños de maternal porque eso } \\
\text { corresponde a su madre. }\end{array}$ & 9 & 30 & 3 & 10 & 3 & 10 & 9 & 30 & 6 & 20 \\
\hline 25 & $\begin{array}{l}\text { Rechazo atender al niño en sus necesidades fisiológicas básicas (pipí, } \\
\text { pupú), porque no corresponde a mi rol de educadora. }\end{array}$ & 9 & 30 & 6 & 20 & 0 & 0 & 3 & 10 & 12 & 40 \\
\hline
\end{tabular}

Nota: Elaboración propia.

En el caso del indicador demostración de afecto (tabla 5) se observa que un 43,3\% están totalmente de acuerdo y de acuerdo con la afirmación "demuestro afecto al niño maternal...para que su adaptación sea satisfactoria”, y un 26,6\% se encuentran indecisos, lo que sugiere que las docentes tienen una disposición un poco más favorable hacia la afectividad con los niños del nivel; como plantea el currículo el eje afectivo es una acción básica del docente en toda la práctica pedagógica para potenciar el aprendizaje infantil (Ministerio de Educación y Deportes, 2005). De la misma manera, existe una actitud más favorable hacia brindar afecto a través del juego con los niños (33,3\% de acuerdo con esta afirmación y $10 \%$ en total acuerdo, ítem 27), y, a dar afecto para favorecer el desarrollo infantil (ítem 28).

\section{Tabla 5}

Respuestas de las docentes en cuanto a la Dimensión Afectiva, Indicador, Demostración de afecto. 


\begin{tabular}{|c|c|c|c|c|c|c|c|c|c|c|c|}
\hline & \multirow[b]{2}{*}{ ÍTEM } & \multicolumn{2}{|r|}{ TDA } & \multicolumn{2}{|c|}{ DA } & \multicolumn{2}{|c|}{ I } & \multicolumn{2}{|c|}{ ED } & \multicolumn{2}{|c|}{$\overline{\text { TED }}$} \\
\hline & & $\mathbf{f}$ & $\%$ & f & $\%$ & f & $\%$ & f & $\%$ & & $\%$ \\
\hline 26 & $\begin{array}{l}\text { Demuestro afecto al niño de maternal a través de abrazos, } \\
\text { besos y caricias para potenciar su desarrollo y estabilidad } \\
\text { emocional }\end{array}$ & 10 & 33,3 & 10 & 33,3 & 5 & 16,6 & 5 & 16,6 & 0 & 0 \\
\hline 27 & $\begin{array}{l}\text { Brindo afecto cuando juego con los niños de edad } \\
\text { maternal para conectarme emocionalmente con ellos y } \\
\text { entender sus necesidades e intereses. }\end{array}$ & 3 & 10 & 10 & 33,3 & 8 & 26,6 & 9 & 30 & 0 & 0 \\
\hline 28 & $\begin{array}{l}\text { Doy afecto a los niños de } 0 \text { a } 3 \text { años, para favorecer su } \\
\text { óptimo desarrollo en su interacción con los objetos y el } \\
\text { mundo que lo rodea. }\end{array}$ & 3 & 10 & 10 & 33,3 & 8 & 26,6 & 9 & 30 & 0 & 0 \\
\hline
\end{tabular}

Nota: Elaboración propia.

\section{Dimensión Conductual}

En la dimensión conductual indicador disposición, las docentes se encuentran en un 50 $\%$ en total desacuerdo con solicitar al directivo le asignen una sección de maternal para el año escolar y solo un $10 \%$ se encuentran en total acuerdo para desempeñarse en el aula con niños de 0 a 3 años. Por otra parte, un 60\% muestra una actitud desfavorable hacia la organización de ambientes de aprendizaje adecuados en la etapa maternal (30\% en desacuerdo y $30 \%$ totalmente en desacuerdo). Asimismo, un $40 \%$ de los docentes (10\% totalmente de acuerdo y $30 \%$ de acuerdo) manifiestan tener disposición para investigar los aspectos relacionados a la atención pedagógica del niño de estas edades, en contraposición con un 50\% (20\% en desacuerdo y un $30 \%$ en total desacuerdo) que manifiestan una actitud más desfavorable.

\section{Tabla 6}

Respuestas de las docentes en cuanto a la Dimensión Conductual, Indicador Disposición.

\begin{tabular}{ccccccccccc}
\multirow{2}{*}{ ÍTEM } & \multicolumn{1}{c}{ TDA } & \multicolumn{1}{c}{ DA } & \multicolumn{1}{c}{ I } & \multicolumn{2}{c}{ ED } & \multicolumn{2}{c}{ TED } \\
\cline { 2 - 9 } & $\mathbf{f}$ & $\boldsymbol{\%}$ & $\mathbf{f}$ & $\boldsymbol{\%}$ & $\mathbf{f}$ & $\boldsymbol{\%}$ & $\mathbf{f}$ & $\boldsymbol{\%}$ & $\mathbf{f}$ & $\mathbf{\%}$ \\
\hline una sección de maternal para trabajar & 3 & 10 & 0 & 0 & 9 & 30 & 3 & 10 & 15 & 50
\end{tabular}

29 Solicito al directivo me asigne una sección de maternal para trabajar periódicamente, de esta manera atender las necesidades de este grupo

30 Demuestro disposición para organizar un ambiente adecuado a los $\begin{array}{llllllllll}6 & 20 & 3 & 10 & 3 & 10 & 9 & 30 & 9 & 30\end{array}$ niños de 0 a 3 años 
31 Tengo disposición en investigar todos los aspectos relacionados sobre

$\begin{array}{llllllllll}3 & 10 & 9 & 30 & 3 & 10 & 6 & 20 & 9 & 30\end{array}$ la atención del niño y niña de 0 a 3 años para fortalecer su desarrollo integral.

Nota: Elaboración propia.

Con respecto a la atención que prodigan las docentes al desempeñarse en el aula maternal (tabla 7) solo un 10\% manifiestan una actitud favorable a planificar actividades específicas que potencien las distintas capacidades del niño, el resto tiene una actitud desfavorable: hay un $30 \%$ de docentes indecisas, $10 \%$ en desacuerdo con esta afirmación y $50 \%$ totalmente en desacuerdo. Acerca de la realización de actividades lúdicas durante el desarrollo de la jornada diaria un $50 \%$ muestra una actitud desfavorable (ED y TED) y un 40 $\%$ una actitud favorable (TDA y DA). La evaluación del niño de maternal también se ve afectada por la actitud de la docente pues el $43 \%$ está totalmente en desacuerdo, lo cual probablemente ocasiona que no se diseñen los instrumentos precisos para llevar a cabo este proceso tan vital en la atención pedagógica de los niños.

\section{Tabla 7}

Respuestas de las docentes en cuanto a la Dimensión Conductual, Indicador Atención.

\begin{tabular}{|c|c|c|c|c|c|}
\hline & TDA & DA & I & ED & TH \\
\hline ÍTEM & f $\%$ & f $\%$ & f $\%$ & f \% & $\mathbf{f}$ \\
\hline
\end{tabular}

32 Planifico actividades para que puedan alcanzar un desarrollo óptimo de sus capacidades físicas, intelectuales, afectivas, sociales y morales.

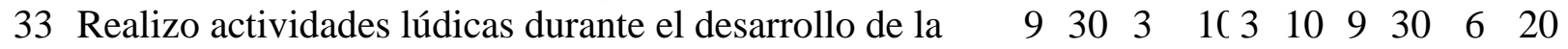
jornada diaria.

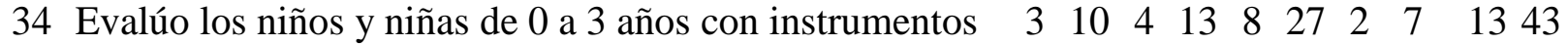
que registren su desarrollo integral.

Nota: Elaboración propia.

\section{CONCLUSIONES}

Al analizar la actitud de las docentes de Educación Inicial hacia la Atención Pedagógica en la Etapa Maternal podemos concluir lo siguiente:

-Las docentes muestran una actitud desfavorable en la dimensión cognitiva, es decir, que desconocen los aspectos más importantes del desarrollo infantil, de las necesidades e 
intereses propios de la edad de 0 a 3 años. Asimismo, manifiestan poca disposición por conocer los fundamentos pedagógicos y psicológicos del aprendizaje en maternal y de las características y organización del ambiente de aprendizaje, a pesar de que son parte de los lineamientos curriculares del Ministerio de Educación (2005).

Al respecto, el MED (2005) establece que el docente es un planificador del ambiente de aprendizaje al tomar en consideración la dimensión física (paredes, espacio, mobiliario); la dimensión funcional (los recursos didácticos y su funcionalidad); la dimensión temporal (organización de la jornada diaria) y la dimensión relacional (interacciones niño-niño, niñodocente, niño-familia, docente-familia), tanto en espacios convencionales como en no convencionales. Asimismo, Ávila (2016) sostiene que la sociedad actual demanda un docente con competencias didácticas, con saberes pedagógicos de alto nivel que le permitan aplicar las estrategias más pertinentes para mediar el aprendizaje en los niños y más aún, un docente que reflexione sobre sus propias prácticas y pueda evaluar sus propios aciertos y desaciertos.

Creemos además, que la especificidad de la etapa maternal requiere de saberes interdisciplinarios para abordar las situaciones pedagógicas con una mirada multidimensional del niño, pues éste posee intereses, necesidades, potencialidades y formas de ser únicas que merecen ser tratadas con dignidad y ser comprendidas de manera no sentenciosa sino formativa.

-Los docentes muestran en la dimensión afectiva una actitud un poco más favorable hacia la demostración de afecto, así como de prodigar encuentros cariñosos con los niños de maternal. Aún cuando hay cierta cantidad de docentes indecisos, el afecto parece ser una acción poderosa en la práctica docente, pues como es sabido, el aula infantil es algo más que un espacio escolar, es lugar de encuentro humano para aprender, conocer y conocerse, interactuar y establecer lazos afectivos con quienes les rodean. Así, que puede verse como positivo la actitud que demuestran las docentes en este sentido. Al respecto, Castro y Morales (2015) refieren que quien origina el ambiente educativo "es el docente, ya que la empatía y el afecto que demuestra permite que los educandos se sientan queridos y puedan construir de mejor manera sus aprendizajes, siendo la relación dialógica la base entre el docente y el estudiantado" (p. 12).

-En la dimensión conductual, se observa una tensión persistente entre una actitud favorable y desfavorable producto de la resistencia de las docentes para asumir las aulas de 
maternal en cada nuevo año escolar, así como para planificar, realizar actividades lúdicas y evaluar en estas edades.

-Se puede establecer unas tensiones categoriales en cada dimensión actitudinal como conclusión de este estudio: cognitivo-desfavorable; afectivo-favorable y conductual-favorable y desfavorable. Estas tensiones permiten inferir que el docente da menor importancia a los conocimientos específicos para la atención pedagógica en maternal, a llevar organizadamente los procesos didácticos como planificar, implementar estrategias y evaluar; en contraposición con su intención de dar una atención afectiva cercana al niño y cónsona con su naturaleza, es decir, parecen suponer que en el hacer docente de la etapa, la afectividad es lo único importante.

-Se abren nuevas interrogantes al respecto, dirigidas a indagar acerca de las representaciones y subjetividades que subyacen a esta actitud de las docentes, así como las condiciones reales del ambiente de aprendizaje de la etapa maternal en distintos centros de Educación Inicial, que pudiera ser un elemento que afecte la práctica docente.

-Se recomienda difundir estos resultados entre los distintos centros de la región y la universidad para hacer un análisis al respecto y sensibilizar tanto a los docentes en ejercicio como en formación acerca de los hallazgos

-Se recomienda realizar talleres formativos y vivenciales con las docentes que laboran en la etapa maternal para ofrecer orientaciones relacionadas con el desarrollo evolutivo del niño y la niña, el aprendizaje, los aportes recientes de las neurociencias y las características del ambiente de aprendizaje, atendiendo sus cuatro dimensiones: Física, Funcional, Temporal y Relacional. Además, encuentros en el que se puedan escuchar sus dudas, sugerencias e inquietudes con respecto a la atención pedagógica en la etapa maternal.

\section{REFERENCIAS}

Amar, J., Palacio, J., Madariaga, C., Abellos, R., De los Reyes, C., De Castro, A., Lewis, S., Martínez, M., Utria, L., Zanello, L., Ferro, J., Jabba, D., Ramos, J. (2016). Infantia. Prácticas de cuidado en la primera infancia. Barranquilla: Editorial Universidad del Norte. https://dialnet.unirioja.es/descarga/libro/695544.pdf

Asamblea General de las Naciones Unidas (1989).Convención Internacional de los Derechos del Niño. https://www.un.org/es/events/childrenday/pdf/derechos.pdf 
Castro M. y Morales, M. (2015). Los ambientes de aula que promueven el aprendizaje, desde la perspectiva de los niños y niñas escolares. Revista Electrónica Educare, 19(3), 1-32. http://dx.doi.org/10.15359/ree.19-3.11

Constitución de la República Bolivariana de Venezuela. (1999). Gaceta Oficial N 5453 (Extraordinario), 24 de Marzo del 2000.

Echenique, A. y Fermín, M. (2011). Atención pedagógica para la etapa maternal: una revisión del proceso de formación de educadores infantiles. Revista de Investigación, 35(72), 143-165. https://www.redalyc.org/articulo.oa?id=376140387007

Fujimoto, G. (2011). El futuro de la educación iberoamericana: ¿Es la no escolarización una alternativa? Revista de Investigación, 35(72), 13-31. https://www.redalyc.org/pdf/3761/376140387001.pdf

González, F. (2019). Análisis socioeducativo de los Simoncitos Comunitarios en la atención no convencional de la educación inicial. Revista EDUCARE UPEL-IPB - Segunda Nueva Etapa 2.0, 23(1), 27-53. https://doi.org/10.46498/reduipb.v23i1.10

Gutiérrez, S. y Ruiz, M. (2018). Impacto de la educación inicial y preescolar en el neurodesarrollo infantil. IE Revista de investigación educativa de la REDIECH, 9(17), 33-51. $\quad$ http://www.scielo.org.mx/scielo.php?script=sci_arttext\&pid=S2448$\underline{85502018000200033}$

Hernández, R; Fernández, C.; Baptista, P. (2003). Metodología de la investigación. México: Mc Graw Hill

Hurtado, I. y Toro, J. (2007). Paradigmas y Métodos de Investigación en tiempos de cambio. Editorial Episteme consultores. Caracas.

Ley Orgánica de Educación, (2009). Gaceta Oficial de la República Bolivariana de Venezuela. 5929 (Extraordinario), Agosto, 15, 2009.

Ministerio de Educación y Deportes (2005). Currículo de Educación Inicial. Caracas: MED.

Molina, M., Sendra, J. y Sierra, C. (2016). Saberes docentes y educación infantil. Notas pedagógicas para la formación inicial. Revista Iberoamericana de Educación, 72, 6584. https://rieoei.org/RIE/article/view/36

Narodowski. M. y Snaider, C. (2017). ¿Bebés en las escuelas? Infancias hiperescolarizadas en una cultura prefigurativa. Revista Latinoamericana de Ciencias Sociales, Niñez y Juventud, 15 (1), 45-57. https://www.redalyc.org/pdf/773/77349627002.pdf 
New Jersey Council For Young Children. (2013). Del nacimiento a los tres años. Normas de aprendizaje temprano-Nueva Jersey. https://www.state.nj.us/education/ece/guide/standards/birth/standardsSP.pdf

Organización de las Naciones Unidas para la Educación, la Ciencia y la Cultura (2007). Bases Sólidas: Atención y Educación de la Primera Infancia. París: UNESCO.

Ospina, V. (2015). El docente del nivel inicial: Retos para la formación profesional y continua. $\begin{array}{lllll}\text { Revista Educación } & y & \text { Humanismo, } & \text { 18(30), }\end{array}$ http://dx.doi.org/10.17081/eduhum.18.30.1325

Paniagua, M. 2016. Marcadores del desarrollo infantil, enfoque Neuropsicopedagógico. Fides Et Ratio, 12, 81-99, Instituto de Investigación en Ciencias Humanas y la Educación, $\begin{array}{llll}\text { Universidad } & \text { La } & \text { Salle }\end{array}$ http://www.scielo.org.bo/pdf/rfer/v12n12/v12n12_a06.pdf

Pares, Y. (2012). Diseño de un plan de Estrategias para la Estimulación del Desarrollo Integral de los Niños y Niñas de 0 a 3 años, dirigido a los docentes del Centro de Educación Inicial Francisco Lazo Martí de Valle de la Pascua, Estado Guárico [Trabajo de Grado de Maestría en Educación Inicial, Universidad Latinoamericana y del Caribe]. https://docplayer.es/28038106-Universidad-latinoamericana-y-del-caribe-ulaccoordinacion-de-postgrado-maestria-en-educacion-inicial.html

Peralta, M. (2007). Estado del Arte sobre pedagogía de la primera infancia (0-3 años) en Latinoamérica y el Caribe. Santiago de Chile: Instituto Internacional de Educación Infantil.

Picco, C. y Soto, C. (2013). Temas de 0 a 3 años. Experiencias de Educación y Cuidado para la Primera Infancia. Argentina: Ministerio de Educación. http://www.bnm.me.gov.ar/giga1/documentos/EL006532.pdf

Pitluk, L. (2014). Un jardín maternal pleno de posibilidades educativas. Educación Inicial Ministerio de Educación del Gobierno de la Ciudad Autónoma de Buenos Aires, https://www.buenosaires.gob.ar/areas/educacion/curricula/Jardin\%20maternal.pdf

Ramírez-Abrahams, P., Patiño-Mora, V. y Gamboa-Vásquez, E. (septiembre-diciembre, 2014). La educación temprana para niños y niñas del nacimiento a los 3 años: Tres perspectivas de análisis. Revista Electrónica Educare, 18(3), 67-90. http://dx.doi.org/10.15359/ree.18-3.5

Rodríguez, A. (2006). Psicología Social. México: Trillas. 
Sabino, C. (2007). El Proceso de Investigación. Editorial Panapo. Caracas

Saldivia, F. (2014). Actitud del docente ante la aplicación de estrategias de integración para el proceso de adaptación en el niño del nivel maternal [Tesis de Grado de Maestría]. Universidad Pedagógica Experimental Libertador (UPEL), Barquisimeto.

Tovar, L., Pastor, R., Lemus, L., Ocón, C. y Ángel M. (2011). El desarrollo de los niños y niñas menores de tres años. Facultad de Psicología de la Universidad Nacional Autónoma de México.

http://www.psicologia.unam.mx/documentos/pdf/publicaciones/Desarrollo_de_ninos_y _ninas_menores_de_3_anos_Tovar_Pastor_Lemus_Ocon_y_Perez.pdf

Zubieta, E. (2005). Valores y Actitudes. https://www.researchgate.net/publication/327043553_VALORES_Y_ACTITUDES 